PROCEEDINGS OF THE

AMERICAN MATHEMATICAL SOCIETY

Volume 140, Number 3, March 2012, Pages 1075-1083

S 0002-9939(2011)10951-1

Article electronically published on July 11, 2011

\title{
A COMBINATORIAL CONSTRUCTION OF SYMPLECTIC EXPANSIONS
}

\author{
YUSUKE KUNO
}

(Communicated by Daniel Ruberman)

\begin{abstract}
The notion of a symplectic expansion directly relates the topology of a surface to formal symplectic geometry. We give a method to construct a symplectic expansion by solving a recurrence formula given in terms of the Baker-Campbell-Hausdorff series.
\end{abstract}

\section{INTRODUCTION}

Let $\Sigma$ be a compact connected oriented surface of genus $g>0$ with one boundary component. Choose a basepoint $*$ on the boundary $\partial \Sigma$ and let $\pi=\pi_{1}(\Sigma, *)$ be the fundamental group of $\Sigma$.

The notion of (generalized) Magnus expansions was introduced by Kawazumi 5] in his study of the mapping class group of a surface. By definition, the mapping class group $\mathcal{M}_{g, 1}$ is the group of homomorphisms of $\Sigma$ fixing $\partial \Sigma$ pointwise, modulo isotopies fixing $\partial \Sigma$ pointwise. The group $\mathcal{M}_{g, 1}$ faithfully acts on $\pi$, a free group of rank $2 g$, and it is known as the theorem of Dehn-Nielsen that $\mathcal{M}_{g, 1}$ is identified with a subgroup of the automorphism group of a free group:

$$
\mathcal{M}_{g, 1}=\{\varphi \in \operatorname{Aut}(\pi) ; \varphi(\zeta)=\zeta\} .
$$

Here, $\zeta \in \pi$ is the element corresponding to the boundary. See $\S 2$. By choosing a Magnus expansion, the completed group ring of $\pi$ (with respect to the augmentation ideal) is identified with the completed tensor algebra generated by the first homology of the surface. In this way we obtain a tensor expression of the action of $\mathcal{M}_{g, 1}$ on $\pi$. From this point of view, Kawazumi obtained extensions of the Johnson homomorphisms $\tau_{k}$ introduced by Johnson [3], 4]. For details, see [5].

Actually the treatment in [5] is on the automorphism group of a free group, rather than the mapping class group. There are infinitely many Magnus expansions, and the arguments in 5 hold for any Magnus expansions. Recently, Massuyeau [11 introduced the notion of symplectic expansions, which are Magnus expansions satisfying a certain kind of boundary condition, which comes from the fact that $\pi$ has a particular element corresponding to the boundary $\partial \Sigma$. Some nice properties of symplectic expansions are clarified by 7 . In particular, it is shown that there is a Lie algebra homomorphism from the Goldman Lie algebra of $\Sigma$

Received by the editors October 2, 2010 and, in revised form, December 14, 2010.

2010 Mathematics Subject Classification. Primary 57N05, 20F34.

The author is supported by JSPS Research Fellowships for Young Scientists (22.4810).

(C)2011 American Mathematical Society 


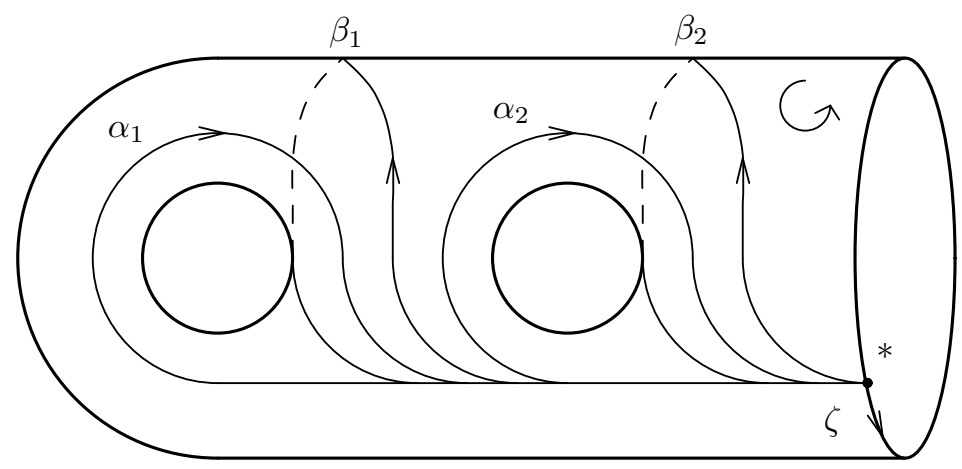

Figure 1. Symplectic generators for $g=2$

(see Goldman [2]) to "associative", one of the three Lie algebras in formal symplectic geometry by Kontsevich [8, via a symplectic expansion (see [7, Theorem 1.2.1).

Although there are infinitely many symplectic expansions (see 7], Proposition 2.8.1), there are not so many known examples. The boundary condition is too strong to be satisfied. For instance, the fatgraph Magnus expansion given by Bene-Kawazumi-Penner [1] is, unfortunately, not symplectic. Kawazumi [6], §6, first constructed an $\mathbb{R}$-valued symplectic expansion, called the harmonic Magnus expansion, by a transcendental method. Massuyeau [11, Proposition 5.6, also gave a $\mathbb{Q}$-valued symplectic expansion using the LMO functor.

The purpose of this paper is to present another construction of symplectic expansions. Our construction is elementary and suitable for computer-aided calculation.

Theorem 1.1. There is an algorithm to construct a symplectic expansion $\theta^{\mathcal{S}}$ associated to any free generating set $\mathcal{S}$ for $\pi$.

It should be remarked here that in the proof of the existence of symplectic expansions (11, Lemma 2.16), Massuyeau already showed how to construct a symplectic expansion degree after degree. Our construction is also inductive, but by using the Dynkin idempotents it fixes the choices that had to be done in the inductive step of [11, Lemma 2.16, hence is canonical. Moreover, our construction works for any free generating set for $\pi$ whereas [11], Lemma 2.16, only deals with symplectic generators.

In $\S 2$, we recall Magnus expansions and symplectic expansions. Theorem 1.1 will be proved in $\S 3$. In $\S 4$, we show a naturality of our construction under the action of a subgroup of $\operatorname{Aut}(\pi)$ including the mapping class group $\mathcal{M}_{g, 1}$. In $\S 5$, we discuss the symplectic expansion associated to symplectic generators.

\section{BASIC NOTIONS}

We denote by $\zeta$ the loop parallel to $\partial \Sigma$ and going in a counterclockwise manner. Explicitly, if we take symplectic generators $\alpha_{1}, \beta_{1}, \ldots, \alpha_{g}, \beta_{g} \in \pi$ as shown in Figure 1. $\zeta=\prod_{i=1}^{g}\left[\alpha_{i}, \beta_{i}\right]$. Here our notation for commutators is $[x, y]:=x y x^{-1} y^{-1}$.

Let $H_{\mathbb{Z}}:=H_{1}(\Sigma ; \mathbb{Z})$ be the first integral homology group of $\Sigma$. We denote $H:=H_{\mathbb{Z}} \otimes_{\mathbb{Z}} \mathbb{Q}$. $H_{\mathbb{Z}}$ is naturally isomorphic to $\pi /[\pi, \pi]$, the abelianization of $\pi$. 
With this identification in mind, we denote $[x]:=x \bmod [\pi, \pi] \in H_{\mathbb{Z}}$, or $[x]:=$ $(x \bmod [\pi, \pi]) \otimes_{\mathbb{Z}} 1 \in H$, for $x \in \pi$.

Let $\widehat{T}$ be the completed tensor algebra generated by $H$. Namely $\widehat{T}=\prod_{m=0}^{\infty} H^{\otimes m}$, where $H^{\otimes m}$ is the tensor space of degree $m$. For each $p \geq 1$, denote $\widehat{T}_{p}:=$ $\prod_{m \geq p}^{\infty} H^{\otimes m}$. Note that the subset $1+\widehat{T}_{1}$ constitutes a subgroup of the multiplicative group of the algebra $\widehat{T}$.

Definition 2.1 (Kawazumi [5]). A map $\theta: \pi \rightarrow 1+\widehat{T}_{1}$ is called a ( $\mathbb{Q}$-valued) Magnus expansion if

(1) $\theta: \pi \rightarrow 1+\widehat{T}_{1}$ is a group homomorphism, and

(2) $\theta(x) \equiv 1+[x] \bmod \widehat{T}_{2}$, for any $x \in \pi$.

The standard Magnus expansion defined by $\theta\left(s_{i}\right)=1+\left[s_{i}\right]$, for some free generating set $\left\{s_{i}\right\}_{i}$ for $\pi$, is the simplest example of a Magnus expansion. This is introduced by Magnus [9] and is often used in combinatorial group theory.

Let $\widehat{\mathcal{L}} \subset \widehat{T}$ be the completed free Lie algebra generated by $H$. The bracket is given by $[u, v]:=u \otimes v-v \otimes u$, and its degree $p$-part $\mathcal{L}_{p}=\widehat{\mathcal{L}} \cap H^{\otimes p}$ is successively given by $\mathcal{L}_{1}=H$ and $\mathcal{L}_{p}=\left[H, \mathcal{L}_{p-1}\right], p \geq 2$. Via the intersection form $(\cdot): H \times$ $H \rightarrow \mathbb{Q}$ on $\Sigma, H$ and its dual $H^{*}=\operatorname{Hom}_{\mathbb{Q}}(H, \mathbb{Q})$ are canonically identified by the map $H \cong H^{*}, X \mapsto(Y \mapsto(Y \cdot X))$. Let $\omega \in \mathcal{L}_{2} \subset H^{\otimes 2}$ be the symplectic form, namely the tensor corresponding to $-1_{H} \in \operatorname{Hom}_{\mathbb{Q}}(H, H)=H^{*} \otimes H=H \otimes H$. Explicitly, if we take symplectic generators as in Figure 1, then $A_{i}=\left[\alpha_{i}\right]$ and $B_{i}=\left[\beta_{i}\right]$ satisfy $\left(A_{i} \cdot B_{j}\right)=-\left(B_{j} \cdot A_{i}\right)=\delta_{i j}$ and $\left(A_{i} \cdot A_{j}\right)=\left(B_{i} \cdot B_{j}\right)=0$; hence we have

$$
\omega=\sum_{i=1}^{g} A_{i} \otimes B_{i}-B_{i} \otimes A_{i}=\sum_{i=1}^{g}\left[A_{i}, B_{i}\right] .
$$

For a Magnus expansion $\theta$, let $\ell^{\theta}:=\log \theta$. Here, $\log$ is the formal power series

$$
\log (x)=\sum_{n=1}^{\infty} \frac{(-1)^{n-1}}{n}(x-1)^{n}
$$

defined on the set $1+\widehat{T}_{1}$. The inverse of $\log$ is given by the exponential $\exp (x)=$ $\sum_{n=0}^{\infty}(1 / n !) x^{n}$. Note that the Baker-Campbell-Hausdorff formula

$$
\begin{aligned}
u \star v:=\log (\exp (u) \exp (v))= & u+v+\frac{1}{2}[u, v]+\frac{1}{12}[u-v,[u, v]] \\
& -\frac{1}{24}[u,[v,[u, v]]]+\cdots
\end{aligned}
$$

endows the underlying set of $\widehat{\mathcal{L}}$ with a group structure. A priori, $\ell^{\theta}$ is a map from $\pi$ to $\widehat{T}_{1}$.

Definition 2.2 (Massuyeau [11]). A Magnus expansion $\theta$ is called symplectic if

(1) $\theta$ is group-like, i.e., $\ell^{\theta}(\pi) \subset \widehat{\mathcal{L}}$, and

(2) $\theta(\zeta)=\exp (\omega)$, or equivalently, $\ell^{\theta}(\zeta)=\omega$.

Remark 2.3. Let $I \pi$ be the augmentation ideal of the group ring $\mathbb{Q} \pi$, and $\widehat{\mathbb{Q}} \pi:=$ $\varliminf_{m} \mathbb{Q} \pi / I \pi^{m}$ the completed group ring of $\pi$. Any Magnus expansion $\theta$ induces an isomorphism $\theta: \widehat{\mathbb{Q}} \pi \stackrel{\cong}{\rightrightarrows} \widehat{T}$ of complete augmented algebras. See [5], Theorem 1.3. Moreover, let $\langle\zeta\rangle$ be the cyclic subgroup of $\pi$ generated by $\zeta$, and $\mathbb{Q}[[\omega]]$ the ring 
of formal power series in the symplectic form $\omega$, which is regarded as a subalgebra of $\widehat{T}$ in an obvious way. Then any symplectic expansion $\theta$ induces an isomorphism $\theta:(\widehat{\mathbb{Q} \pi}, \widehat{\mathbb{Q}\langle\zeta\rangle}) \rightarrow(\widehat{T}, \mathbb{Q}[[\omega]])$ of complete Hopf algebras. See [7], $\S 6.2$.

\section{Main CONSTRUCtion}

We fix a free generating set $\mathcal{S}=\left\{s_{1}, \ldots, s_{2 g}\right\}$ for $\pi$. We denote $S_{i}:=\left[s_{i}\right] \in H$, $1 \leq i \leq 2 g$. Let $x_{1} x_{2} \cdots x_{p}$ be the unique reduced word in $\mathcal{S}$ representing $\zeta$.

Definition 3.1. Fix an integer $n \geq 1$. A set $\left\{\ell_{j}\left(s_{i}\right): 1 \leq i \leq 2 g, 1 \leq j \leq n\right\} \subset \widehat{\mathcal{L}}$ is called a partial symplectic expansion up to degree $n$ if

(1) $\ell_{1}\left(s_{i}\right)=S_{i}$, for $1 \leq i \leq 2 g$,

(2) $\ell_{j}\left(s_{i}\right) \in \mathcal{L}_{j}$, for $1 \leq i \leq 2 g, 1 \leq j \leq n$, and

(3) if we set $\bar{\ell}_{n}\left(s_{i}\right)=\sum_{j=1}^{n} \ell_{j}\left(s_{i}\right)$ for $1 \leq i \leq 2 g$, then

$$
\bar{\ell}_{n}\left(x_{1}\right) \star \bar{\ell}_{n}\left(x_{2}\right) \star \cdots \star \bar{\ell}_{n}\left(x_{p}\right) \equiv \omega \bmod \widehat{T}_{n+2}
$$

Here, we understand $\bar{\ell}_{n}\left(s_{i}^{-1}\right)=-\bar{\ell}_{n}\left(s_{i}\right)$.

This notion could be thought of as an approximation to a symplectic expansion. In this section we give a method to refine an approximation up to degree $n-1$, to the one up to degree $n$. Repeating this process, we will obtain a symplectic expansion.

We need two lemmas.

Lemma 3.2. Suppose $4 g$ elements $Y_{1}, \ldots, Y_{2 g}, Z_{1}, \ldots, Z_{2 g} \in H$ satisfy $\sum_{i=1}^{2 g} Y_{i} \otimes$ $Z_{i}=\omega \in H^{\otimes 2}$. Then $Z_{1}, \ldots, Z_{2 g}$ constitute a basis for $H$.

Proof. Since $\omega$ corresponds to $-1_{H} \in \operatorname{Hom}_{\mathbb{Q}}(H, H)$ (see $\S 2$ ), for any $X \in H$, we have

$$
X=\omega(-X)=\sum_{i=1}^{2 g}\left(-X \cdot Y_{i}\right) Z_{i} .
$$

This shows that the $2 g$ elements $Z_{1}, \ldots, Z_{2 g}$ generate $H$. This proves the lemma.

Since $\pi$ is free, the quotient $[\pi, \pi] /[\pi,[\pi, \pi]]$ is naturally isomorphic to $\Lambda^{2} H_{\mathbb{Z}}$, the second exterior product of $H_{\mathbb{Z}}$. The isomorphism is induced by the homomorphism $f:[\pi, \pi] \rightarrow \Lambda^{2} H_{\mathbb{Z}}$ which maps the commutator $[x, y]$ to $[x] \wedge[y]$. Note that $\Lambda^{2} H_{\mathbb{Z}}$ is naturally identified with a subgroup of $H^{\otimes 2}$ by

$$
\Lambda^{2} H_{\mathbb{Z}} \rightarrow H^{\otimes 2}, X \wedge Y \mapsto X \otimes Y-Y \otimes X,
$$

and under this identification we have $f(\zeta)=\omega$.

Lemma 3.3. Let $y_{1} \cdots y_{q}$ be a word in $\mathcal{S}$ and suppose $y_{1} \cdots y_{q}$ lies in the commutator subgroup $[\pi, \pi]$. Then

$$
f\left(y_{1} \cdots y_{q}\right)=\frac{1}{2} \sum_{i<j}\left[y_{i}\right] \wedge\left[y_{j}\right] .
$$

Proof. We may assume $q \geq 2$. We prove the lemma by induction on $q$. The case $q=2$ is trivial. Suppose $q>2$. Then there must exist $i \geq 1$ such that $y_{i+1}=y_{1}^{-1}$, and

$$
y_{1} \cdots y_{q}=y_{1} y_{2} \cdots y_{i} y_{1}^{-1} y_{i+2} \cdots y_{q}=\left[y_{1}, y_{2} \cdots y_{i}\right] y_{2} \cdots y_{i} y_{i+2} \cdots y_{q}
$$


Hence $f\left(y_{1} \cdots y_{q}\right)=f\left(\left[y_{1}, y_{2} \cdots y_{i}\right]\right)+f\left(y_{2} \cdots y_{i} y_{i+2} \cdots y_{q}\right)$. The first term equals

$$
\left[y_{1}\right] \wedge\left(\left[y_{2}\right]+\cdots+\left[y_{i}\right]\right)=\frac{1}{2}\left(\left[y_{1}\right] \wedge\left(\left[y_{2}\right]+\cdots+\left[y_{i}\right]\right)+\left(\left[y_{2}\right]+\cdots+\left[y_{i}\right]\right) \wedge\left[y_{i+1}\right]\right)
$$

since $\left[y_{1}\right]=-\left[y_{i+1}\right]$, and the second term equals

$$
\frac{1}{2} \sum_{\substack{k<\ell ; \\ k, \ell \neq 1, i+1}}\left[y_{k}\right] \wedge\left[y_{\ell}\right],
$$

by the inductive assumption. This proves the lemma.

Let $\Phi: \widehat{T}_{1} \rightarrow \widehat{\mathcal{L}}$ be the linear map defined by $\Phi\left(Y_{1} \otimes \cdots \otimes Y_{m}\right)=\left[Y_{1},\left[\cdots\left[Y_{m-1}\right.\right.\right.$, $\left.\left.\left.Y_{m}\right] \cdots\right]\right], Y_{i} \in H, m \geq 1$. We have $\Phi(u)=m u$ and $\Phi(u v)=[u, \Phi(v)]$ for any $u \in \mathcal{L}_{m}, v \in \widehat{T}_{1}$. See Serre [12], Part I, Theorem 8.1, p. 28. The maps $\left.(1 / m) \Phi\right|_{H^{\otimes m}}$ are called the Dynkin idempotents. From these two properties we see that the restriction of the map

$$
\frac{1}{m+1}(\mathrm{id} \otimes \Phi): H^{\otimes m+1} \rightarrow H \otimes \mathcal{L}_{m}
$$

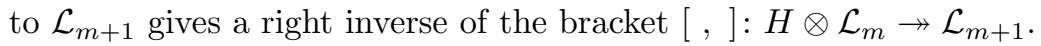

Let $n \geq 2$ and let $\left\{\ell_{j}\left(s_{i}\right): 1 \leq j \leq n-1,1 \leq i \leq 2 g\right\}$ be a partial symplectic expansion up to degree $n-1$. We have

$$
\bar{\ell}_{n-1}\left(x_{1}\right) \star \bar{\ell}_{n-1}\left(x_{2}\right) \star \cdots \star \bar{\ell}_{n-1}\left(x_{p}\right) \equiv \omega \bmod \widehat{T}_{n+1} .
$$

Let $V_{n+1} \in \mathcal{L}_{n+1}$ be the degree $(n+1)$-part of $\bar{\ell}_{n-1}\left(x_{1}\right) \star \bar{\ell}_{n-1}\left(x_{2}\right) \star \cdots \star \bar{\ell}_{n-1}\left(x_{p}\right)$. By Lemma 3.3 we have $\omega=f(\zeta)=f\left(x_{1} \cdots x_{p}\right)=\frac{1}{2} \sum_{i<j} X_{i} \wedge X_{j}=\frac{1}{2} \sum_{i<j}\left(X_{i} \otimes\right.$ $\left.X_{j}-X_{j} \otimes X_{i}\right)$, where $X_{i}=\left[x_{i}\right]$. Since $S_{1}, \ldots, S_{2 g}$ constitute a basis for $H$, we can uniquely write

$$
\omega=\frac{1}{2} \sum_{i<j}\left(X_{i} \otimes X_{j}-X_{j} \otimes X_{i}\right)=\sum_{i=1}^{2 g} S_{i} \otimes Z_{i}, \quad \text { where } Z_{i}=\sum_{k} c_{i k} S_{k}, \quad c_{i k} \in \mathbb{Z} .
$$

Also, in view of applying (3.2) we write $V_{n+1} \in \mathcal{L}_{n+1} \subset H^{\otimes n+1}$ as

$$
V_{n+1}=\sum_{i=1}^{2 g} S_{i} \otimes V_{n}^{S_{i}}, \quad V_{n}^{S_{i}} \in H^{\otimes n}
$$

Now by Lemma $3.2 Z_{1}, \ldots, Z_{2 g}$ constitute a basis for $H$; hence the matrix $\left\{c_{i k}\right\}_{i, k}$ is of full rank. Let $\left\{d_{i k}\right\}_{i, k}$ be the inverse matrix of $\left\{c_{i k}\right\}_{i, k}$.

Proposition 3.4. Keep the same notation as above. Set $W_{i}:=(-1 /(n+1)) \Phi\left(V_{n}^{S_{i}}\right)$ $\in \mathcal{L}_{n}$ for $1 \leq i \leq 2 g$, and $\ell_{n}\left(s_{i}\right):=\sum_{k} d_{i k} W_{k}$ for $1 \leq i \leq 2 g$. Then $\left\{\ell_{j}\left(s_{i}\right): 1 \leq\right.$ $j \leq n-1,1 \leq i \leq 2 g\} \cup\left\{\ell_{n}\left(s_{i}\right): 1 \leq i \leq 2 g\right\}$ is a partial symplectic expansion up to degree $n$.

Proof. Set $\bar{\ell}_{n}\left(s_{i}\right)=\bar{\ell}_{n-1}\left(s_{i}\right)+\ell_{n}\left(s_{i}\right)$. Understanding $\ell_{n}\left(s_{i}^{-1}\right)=-\ell_{n}\left(s_{i}\right)$, we have $\sum_{i=1}^{p} \ell_{n}\left(x_{i}\right)=0$ since $\zeta \in[\pi, \pi]$. Hence we have $\bar{\ell}_{n}\left(x_{1}\right) \star \bar{\ell}_{n}\left(x_{2}\right) \star \cdots \star \bar{\ell}_{n}\left(x_{p}\right) \equiv$ $\omega \bmod \widehat{T}_{n+1}$ from (3.3). By (2.2) we see that the degree $(n+1)$-part of $\bar{\ell}_{n}\left(x_{1}\right) \star$ $\bar{\ell}_{n}\left(x_{2}\right) \star \cdots \star \bar{\ell}_{n}\left(x_{p}\right)$ equals

$$
V_{n+1}+\frac{1}{2} \sum_{i<j}\left(\left[X_{i}, \ell_{n}\left(x_{j}\right)\right]+\left[\ell_{n}\left(x_{i}\right), X_{j}\right]\right) .
$$


Let $\lambda: H \rightarrow \mathcal{L}_{n}$ be the linear map defined by $\lambda\left(S_{i}\right)=\ell_{n}\left(s_{i}\right)$ and apply the linear map [id, $\lambda]: H^{\otimes 2} \rightarrow H^{\otimes n+1}$ to (3.4). Then we obtain

$$
\frac{1}{2} \sum_{i<j}\left(\left[X_{i}, \ell_{n}\left(x_{j}\right)\right]-\left[X_{j}, \ell_{n}\left(x_{i}\right)\right]\right)=\sum_{i=1}^{2 g}\left[S_{i}, W_{i}^{\prime}\right], \quad W_{i}^{\prime}=\sum_{k} c_{i k} \ell_{n}\left(s_{k}\right) .
$$

But $W_{i}^{\prime}=\sum_{k} \sum_{j} c_{i k} d_{k j} W_{j}=W_{i}$. Hence (3.5) is equal to

$$
V_{n+1}+\sum_{i=1}^{2 g}\left[S_{i}, W_{i}\right]=V_{n+1}-\frac{1}{n+1} \sum_{i=1}^{2 g}\left[S_{i}, \Phi\left(V_{n}^{S_{i}}\right)\right]=V_{n+1}-\frac{1}{n+1} \Phi\left(V_{n+1}\right)=0,
$$

since $V_{n+1} \in \mathcal{L}_{n+1}$. Therefore, we have $\bar{\ell}_{n}\left(x_{1}\right) \star \bar{\ell}_{n}\left(x_{2}\right) \star \cdots \star \bar{\ell}_{n}\left(x_{p}\right) \equiv \omega \bmod \widehat{T}_{n+2}$. This completes the proof.

We can now conclude the proof of Theorem 1.1. Denote $\mathcal{S}=\left\{s_{1}, \ldots, s_{2 g}\right\}$ and set $\ell_{1}\left(s_{i}\right):=S_{i}, 1 \leq i \leq 2 g$. By the Baker-Campbell-Hausdorff formula (2.2) and Lemma 3.3. $\left\{\ell_{1}\left(s_{i}\right)\right\}_{1 \leq i \leq 2 g}$ is a partial symplectic expansion up to degree 1 . Applying Proposition 3.4, we obtain $\left\{\ell_{j}\left(s_{i}\right) ; 1 \leq i \leq 2 g, j \geq 1\right\}$ satisfying (3.1) for any $n \geq 1$. Setting $\ell^{\mathcal{S}}\left(s_{i}\right):=\sum_{j=1}^{\infty} \ell_{j}\left(s_{i}\right) \in \widehat{\mathcal{L}}$ and $\theta^{\mathcal{S}}\left(s_{i}\right):=\exp \left(\ell^{\mathcal{S}}\left(s_{i}\right)\right)$, we extend $\theta^{\mathcal{S}}$ to a homomorphism from $\pi$ using the universality of the free group $\pi$. Then $\theta^{\mathcal{S}}$ is the desired symplectic expansion. Note that the result $\theta^{\mathcal{S}}$ does not depend on the total ordering on the set $\mathcal{S}$. This completes the proof of Theorem 1.1.

Remark 3.5. For a group-like expansion $\theta$, we denote $\ell^{\theta}(x)=\sum_{j=1}^{\infty} \ell_{j}^{\theta}(x), \ell_{j}^{\theta}(x) \in$ $\mathcal{L}_{j}$, for $x \in \pi$. Proposition 3.4 can be phrased shortly as: a choice of a free generating set for $\pi$ gives a canonical way of modifying any group-like expansion $\theta$ satisfying $\ell^{\theta}(\zeta) \equiv \omega \bmod \widehat{T}_{n+1}$ for some $n \geq 2$ into a group-like expansion satisfying the same congruence with $n+1$ replaced by $n+2$, without changing $\ell_{j}^{\theta}(x)$, for $1 \leq j \leq n-1$.

\section{NATURALity}

Let $\operatorname{Aut}(\pi)$ be the automorphism group of $\pi$. For $\varphi \in \operatorname{Aut}(\pi)$, let $|\varphi|$ be the filter-preserving algebra automorphism of $\widehat{T}$ induced by the action of $\varphi$ on the first homology $H$. If $\theta$ is a Magnus expansion, then the composite $|\varphi| \circ \theta \circ \varphi^{-1}$ is again a Magnus expansion.

We show a naturality of the symplectic expansion $\theta^{\mathcal{S}}$ given in Theorem 1.1. Note that fatgraph Magnus expansions have a similar property (see [1], Theorem 4.2).

Proposition 4.1. Suppose $\varphi \in \operatorname{Aut}(\pi)$ satisfies $\varphi(\zeta)=\zeta$, or $\varphi(\zeta)=\zeta^{-1}$. Then

$$
\theta^{\varphi(\mathcal{S})}=|\varphi| \circ \theta^{\mathcal{S}} \circ \varphi^{-1} .
$$

Proof. Let $\mathcal{S}=\left\{s_{1}, \ldots, s_{2 g}\right\}$. We shall put $\mathcal{S}$ on the upper right of the objects $V_{n+1}, \ell_{j}, c_{i k}$, etc., in the proof of Proposition 3.4 to indicate their dependence on $\mathcal{S}$.

The equality we are going to prove is equivalent to $\ell^{\varphi(\mathcal{S})}\left(\varphi\left(s_{i}\right)\right)=|\varphi| \ell^{\mathcal{S}}\left(s_{i}\right)$, or, $\ell_{n}^{\varphi(\mathcal{S})}\left(\varphi\left(s_{i}\right)\right)=|\varphi| \ell_{n}^{\mathcal{S}}\left(s_{i}\right)$ for any $n \geq 1$. We prove this by induction on $n$. Since $\ell_{1}^{\varphi(\mathcal{S})}\left(\varphi\left(s_{i}\right)\right)=\left[\varphi\left(s_{i}\right)\right]=|\varphi|\left[s_{i}\right]$, the case $n=1$ is clear. Suppose $n \geq 2$.

First we assume $\varphi(\zeta)=\zeta$. Then $\varphi\left(x_{1}\right) \cdots \varphi\left(x_{p}\right)$ is a word in $\varphi(\mathcal{S})$ representing $\zeta$, and we have $|\varphi| \omega=\omega$ since $\varphi(\zeta)=\zeta$ and the homomorphism $f:[\pi, \pi] \rightarrow \Lambda^{2} H_{\mathbb{Z}}$ 
in $\S 3$ is $\operatorname{Aut}(\pi)$-equivariant. By the inductive assumption, we have $\bar{\ell}_{n-1}^{\varphi(\mathcal{S})}\left(\varphi\left(s_{i}\right)\right)=$ $|\varphi| \bar{\ell}_{n-1}^{\mathcal{S}}\left(s_{i}\right)$; hence applying $|\varphi|$ to the congruence $\bar{\ell}_{n-1}^{\mathcal{S}}\left(x_{1}\right) \star \bar{\ell}_{n-1}^{\mathcal{S}}\left(x_{2}\right) \star \cdots \star \bar{\ell}_{n-1}^{\mathcal{S}}\left(x_{p}\right) \equiv$ $\omega+V_{n+1}^{\mathcal{S}} \bmod \widehat{T}_{n+2}$, we obtain $V_{n+1}^{\varphi(\mathcal{S})}=|\varphi| V_{n+1}^{\mathcal{S}}$. Therefore, writing $V_{n+1}^{\varphi(\mathcal{S})}=$ $\sum_{i=1}^{2 g}\left(|\varphi| S_{i}\right) \otimes V_{n}^{|\varphi| S_{i}}$, we have $V_{n}^{|\varphi| S_{i}}=|\varphi| V_{n}^{S_{i}}$.

On the other hand, applying $|\varphi|$ to (3.4), we obtain

$$
\omega=\sum_{i=1}^{2 g}|\varphi| S_{i} \otimes Z_{i}^{\varphi(\mathcal{S})}, \quad Z_{i}^{\varphi(\mathcal{S})}=\sum_{k} c_{i k}|\varphi| S_{k} .
$$

This implies that $c_{i k}^{\varphi(\mathcal{S})}=c_{i k}^{\mathcal{S}}$; hence $d_{i k}^{\varphi(\mathcal{S})}=d_{i k}^{\mathcal{S}}$. We conclude that $W_{i}^{\varphi(\mathcal{S})}=|\varphi| W_{i}^{\mathcal{S}}$ and $\ell_{n}^{\varphi(\mathcal{S})}\left(\varphi\left(s_{i}\right)\right)=|\varphi| \ell_{n}^{\mathcal{S}}\left(s_{i}\right)$, as desired.

If $\varphi(\zeta)=\zeta^{-1}$, the same argument shows that $V_{n+1}^{\varphi(\mathcal{S})}=-|\varphi| V_{n+1}^{\mathcal{S}}$ and $c_{i k}^{\varphi(\mathcal{S})}=$ $-c_{i k}^{\mathcal{S}}$ because in this case $\varphi\left(x_{1}\right) \cdots \varphi\left(x_{p}\right)$ is a word in $\varphi(\mathcal{S})$ representing $\zeta^{-1}$, and we have $|\varphi| \omega=-\omega$. Hence we again obtain $\ell_{n}^{\varphi(\mathcal{S})}\left(\varphi\left(s_{i}\right)\right)=|\varphi| \ell_{n}^{\mathcal{S}}\left(s_{i}\right)$. This completes the induction.

\section{Symplectic Generators}

Let $\mathcal{S}_{0}=\left\{\alpha_{1}, \beta_{1}, \ldots, \alpha_{g}, \beta_{g}\right\}$ be symplectic generators as in $\S 2$, and let $\theta^{0}=\theta^{\mathcal{S}_{0}}$ be the symplectic expansion associated to $\mathcal{S}_{0}$, given by the algorithm of Theorem 1.1. For simplicity we write $\alpha_{1}, \beta_{1}, \ldots, \alpha_{g}, \beta_{g}=\xi_{1}, \ldots, \xi_{2 g}$. Let $T \in \operatorname{Aut}(\pi)$ be the automorphism defined by $T\left(\xi_{i}\right)=\xi_{2 g+1-i}, 1 \leq i \leq 2 g$. Then we have $T(\zeta)=\zeta^{-1}$ and $T\left(\mathcal{S}^{0}\right)=\mathcal{S}^{0}$. By Proposition 4.1, we obtain a certain kind of symmetry for $\theta^{0}$.

Proposition 5.1. Let $\theta^{0}$ be the symplectic expansion as above. Then

$$
\theta^{0}\left(\xi_{2 g+1-i}\right)=|T| \theta^{0}\left(\xi_{i}\right), \quad 1 \leq i \leq 2 g .
$$

Finally, we give a more explicit formula for $\ell^{\mathcal{S}_{0}}$ in a form suitable for computeraided calculation. First we give another description of $V_{n+1}$ which does not involve the Baker-Campbell-Hausdorff series. Let $n \geq 2$ and let $\left\{\ell_{j}\left(s_{i}\right): 1 \leq j \leq n-1,1 \leq\right.$ $i \leq 2 g\}$ be a partial symplectic expansion up to degree $n-1$. Set $\bar{\theta}_{n-1}\left(s_{i}\right):=$ $\exp \left(\bar{\ell}_{n-1}\left(s_{i}\right)\right)$ and $\bar{\theta}_{n-1}\left(s_{i}^{-1}\right):=\exp \left(-\bar{\ell}_{n-1}\left(s_{i}\right)\right)$. From (3.3), we have $\bar{\ell}_{n-1}\left(x_{1}\right) \star$ $\bar{\ell}_{n-1}\left(x_{2}\right) \star \cdots \star \bar{\ell}_{n-1}\left(x_{p}\right) \equiv \omega+V_{n+1} \bmod \widehat{T}_{n+2}$. Applying the exponential, we obtain $\bar{\theta}_{n-1}\left(x_{1}\right) \bar{\theta}_{n-1}\left(x_{2}\right) \cdots \bar{\theta}_{n-1}\left(x_{p}\right) \equiv \exp (\omega)+V_{n+1} \bmod \widehat{T}_{n+2}$. Hence

$$
V_{n+1}=\left(\bar{\theta}_{n-1}\left(x_{1}\right) \bar{\theta}_{n-1}\left(x_{2}\right) \cdots \bar{\theta}_{n-1}\left(x_{p}\right)-\exp (\omega)\right)_{n+1},
$$

where the subscript $n+1$ in the right-hand side means taking the degree $(n+1)$-part.

Let us consider the case $\mathcal{S}=\mathcal{S}_{0}$. Then $\zeta=\prod_{i=1}^{g}\left[\alpha_{i}, \beta_{i}\right]$. For $X, Y \in \widehat{T}_{1}$, by a direct computation, we have

$$
(1+X)(1+Y)(1+X)^{-1}(1+Y)^{-1}=1+\sum_{i, j \geq 0}(-1)^{i+j}[X, Y] X^{i} Y^{j} .
$$

See Magnus-Karrass-Solitar [10, $\S 5.5,(7 \mathrm{a})$ for a similar formula. Therefore in case $\mathcal{S}=\mathcal{S}_{0}$, (5.1) becomes

$$
V_{n+1}=\left(\prod_{i=1}^{g} G\left(\bar{\theta}_{n-1}\left(\alpha_{i}\right)-1, \bar{\theta}_{n-1}\left(\beta_{i}\right)-1\right)-\exp (\omega)\right)_{n+1},
$$


where $G(X, Y)$ is the right-hand side of (5.2). From (2.1) and (3.4), we obtain the following recursive formulas for $\ell^{\mathcal{S}_{0}}$ :

$$
\begin{aligned}
& \ell_{n}^{\mathcal{S}_{0}}\left(\alpha_{i}\right)=\frac{1}{n+1} \Phi\left(V_{n}^{B_{i}}\right), \\
& \ell_{n}^{\mathcal{S}_{0}}\left(\beta_{i}\right)=\frac{-1}{n+1} \Phi\left(V_{n}^{A_{i}}\right) .
\end{aligned}
$$

In this way we can effectively compute the terms of $\ell^{\mathcal{S}_{0}}\left(\xi_{i}\right)$. Here we give the first few terms of $\ell^{\mathcal{S}_{0}}$ for $g=1,2$.

Example 5.2 (the case of genus 1). For simplicity, we write $\alpha_{1}=\alpha, \beta_{1}=\beta$ and $A_{1}=A, B_{1}=B$. Modulo $\widehat{T}_{6}$, we have

$$
\begin{aligned}
\ell^{\mathcal{S}_{0}}(\alpha) \equiv & A+\frac{1}{2}[A, B]+\frac{1}{12}[B,[B, A]]-\frac{1}{8}[A,[A, B]]+\frac{1}{24}[A,[A,[A, B]]] \\
& -\frac{1}{720}[B,[B,[B,[B, A]]]]-\frac{1}{288}[A,[A,[A,[A, B]]]]-\frac{1}{288}[A,[B,[B,[B, A]]]] \\
& -\frac{1}{288}[B,[A,[A,[A, B]]]]+\frac{1}{144}[[A, B],[B,[B, A]]]+\frac{1}{128}[[A, B],[A,[A, B]]] ; \\
\ell^{\mathcal{S}_{0}}(\beta) \equiv & B-\frac{1}{2}[A, B]+\frac{1}{12}[A,[A, B]]-\frac{1}{8}[B,[B, A]]+\frac{1}{24}[B,[B,[B, A]]] \\
& -\frac{1}{720}[A,[A,[A,[A, B]]]]-\frac{1}{288}[B,[B,[B,[B, A]]]]-\frac{1}{288}[B,[A,[A,[A, B]]]] \\
& -\frac{1}{288}[A,[B,[B,[B, A]]]]-\frac{1}{144}[[A, B],[A,[A, B]]]-\frac{1}{128}[[A, B],[B,[B, A]]] .
\end{aligned}
$$

Example 5.3 (the case of genus 2). Modulo $\widehat{T}_{5}$, we have

$$
\begin{aligned}
\ell^{\mathcal{S}_{0}}\left(\alpha_{1}\right) \equiv & A_{1}+\frac{1}{2}\left[A_{1}, B_{1}\right] \\
& +\frac{1}{12}\left[B_{1},\left[B_{1}, A_{1}\right]\right]-\frac{1}{8}\left[A_{1},\left[A_{1}, B_{1}\right]\right]-\frac{1}{4}\left[A_{1},\left[A_{2}, B_{2}\right]\right] \\
& +\frac{1}{24}\left[A_{1},\left[A_{1},\left[A_{1}, B_{1}\right]\right]\right]-\frac{1}{10}\left[\left[A_{1}, B_{1}\right],\left[A_{2}, B_{2}\right]\right]+\frac{1}{40}\left[A_{1},\left[B_{1},\left[A_{2}, B_{2}\right]\right]\right] \\
& +\frac{1}{40}\left[A_{1},\left[B_{2},\left[A_{2}, B_{2}\right]\right]\right]+\frac{1}{40}\left[A_{1},\left[A_{1},\left[A_{2}, B_{2}\right]\right]\right]+\frac{1}{40}\left[A_{1},\left[A_{2},\left[A_{2}, B_{2}\right]\right]\right] ; \\
\ell^{\mathcal{S}_{0}}\left(\beta_{1}\right) \equiv & B_{1}-\frac{1}{2}\left[A_{1}, B_{1}\right] \\
& +\frac{1}{12}\left[A_{1},\left[A_{1}, B_{1}\right]\right]-\frac{1}{8}\left[B_{1},\left[B_{1}, A_{1}\right]\right]-\frac{1}{4}\left[B_{1},\left[A_{2}, B_{2}\right]\right] \\
& +\frac{1}{24}\left[B_{1},\left[B_{1},\left[B_{1}, A_{1}\right]\right]\right]+\frac{1}{10}\left[\left[A_{1}, B_{1}\right],\left[A_{2}, B_{2}\right]\right]+\frac{1}{40}\left[B_{1},\left[A_{1},\left[A_{2}, B_{2}\right]\right]\right] \\
& +\frac{1}{40}\left[B_{1},\left[A_{2},\left[A_{2}, B_{2}\right]\right]\right]+\frac{1}{40}\left[B_{1},\left[B_{1},\left[A_{2}, B_{2}\right]\right]\right]+\frac{1}{40}\left[B_{1},\left[B_{2},\left[A_{2}, B_{2}\right]\right]\right] ; \\
\ell^{\mathcal{S}_{0}}\left(\alpha_{2}\right) \equiv & A_{2}+\frac{1}{2}\left[A_{2}, B_{2}\right] \\
& +\frac{1}{12}\left[B_{2},\left[B_{2}, A_{2}\right]\right]-\frac{1}{8}\left[A_{2},\left[A_{2}, B_{2}\right]\right]+\frac{1}{4}\left[A_{2},\left[A_{1}, B_{1}\right]\right] \\
& +\frac{1}{24}\left[A_{2},\left[A_{2},\left[A_{2}, B_{2}\right]\right]\right]-\frac{1}{10}\left[\left[A_{1}, B_{1}\right],\left[A_{2}, B_{2}\right]\right]-\frac{1}{40}\left[A_{2},\left[B_{2},\left[A_{1}, B_{1}\right]\right]\right] \\
& -\frac{1}{40}\left[A_{2},\left[B_{1},\left[A_{1}, B_{1}\right]\right]\right]-\frac{1}{40}\left[A_{2},\left[A_{2},\left[A_{1}, B_{1}\right]\right]\right]-\frac{1}{40}\left[A_{2},\left[A_{1},\left[A_{1}, B_{1}\right]\right]\right] ;
\end{aligned}
$$




$$
\begin{aligned}
\ell^{\mathcal{S}_{0}}\left(\beta_{2}\right) \equiv & B_{2}-\frac{1}{2}\left[A_{2}, B_{2}\right] \\
& +\frac{1}{12}\left[A_{2},\left[A_{2}, B_{2}\right]\right]-\frac{1}{8}\left[B_{2},\left[B_{2}, A_{2}\right]\right]+\frac{1}{4}\left[B_{2},\left[A_{1}, B_{1}\right]\right] \\
& +\frac{1}{24}\left[B_{2},\left[B_{2},\left[B_{2}, A_{2}\right]\right]\right]+\frac{1}{10}\left[\left[A_{1}, B_{1}\right],\left[A_{2}, B_{2}\right]\right]-\frac{1}{40}\left[B_{2},\left[A_{2},\left[A_{1}, B_{1}\right]\right]\right] \\
& -\frac{1}{40}\left[B_{2},\left[A_{1},\left[A_{1}, B_{1}\right]\right]\right]-\frac{1}{40}\left[B_{2},\left[B_{2},\left[A_{1}, B_{1}\right]\right]\right]-\frac{1}{40}\left[B_{2},\left[B_{1},\left[A_{1}, B_{1}\right]\right]\right] .
\end{aligned}
$$

\section{ACKNOWLEDGMENTS}

The author wishes to express his gratitude to Alex Bene, who kindly suggested to him to extend the construction for not necessarily symplectic generators; Nariya Kawazumi for communicating to him a proof of a symmetry property of $\theta^{0}$; and Robert Penner for warm comments on a rough draft of this paper. He also would like to thank Shigeyuki Morita and Masatoshi Sato for valuable comments.

\section{REFERENCES}

1. A. J. Bene, N. Kawazumi, and R. C. Penner, Canonical extensions of the Johnson homomorphisms to the Torelli groupoid, Adv. Math. 221, 627-659 (2009). MR2508933 (2010b:32015)

2. W. M. Goldman, Invariant functions on Lie groups and Hamiltonian flows of surface group representations, Invent. Math. 85, 263-302 (1986). MR846929 (87j:32069)

3. D. Johnson, An abelian quotient of the mapping class group $\mathcal{I}_{g}$, Math. Ann. 249, 225-242 (1980). MR 579103(82a:57008)

4. D. Johnson, A survey of the Torelli group, Contemporary Math. 20, 165-179, Amer. Math. Soc., Providence, RI (1983). MR718141 (85d:57009)

5. N. Kawazumi, Cohomological aspects of Magnus expansions, preprint, math.GT/0505497 (2005).

6. N. Kawazumi, Harmonic Magnus expansion on the universal family of Riemann surfaces, preprint, math.GT/0603158 (2006).

7. N. Kawazumi and Y. Kuno, The logarithms of Dehn twists, preprint, arXiv:1008.5017 (2010).

8. M. Kontsevich, Formal (non)-commutative symplectic geometry, in: "The Gel'fand Mathematical Seminars, 1990-1992", Birkhäuser, Boston, 173-187 (1993). MR.1247289(94i:58212)

9. W. Magnus, Beziehungen zwischen Gruppen und Idealen in einem speziellen Ring, Math. Ann. 111, 259-280 (1935). MR1512992

10. W. Magnus, A. Karrass, and D. Solitar, Combinatorial group theory, Dover, New York (1976). MR 0422434 (54:10423)

11. G. Massuyeau, Infinitesimal Morita homomorphisms and the tree-level of the LMO invariant, preprint, arXiv:0809.4629 (2008).

12. J. -P. Serre, Lie algebras and Lie groups, Lecture Notes in Mathematics 1500, Springer-Verlag, Berlin (2006). MR2179691 (2006e:17001)

Department of Mathematics, Graduate School of Science, Hiroshima University, 1-3-1 Kagamiyama, Higashi-Hiroshima, Hiroshima 739-8526, Japan

E-mail address: kunotti@hiroshima-u.ac.jp 